\title{
Stochastic regime switching SIS epidemic model with vaccination driven by Lévy noise
}

Yingjia Guo*

\section{*Correspondence:} guo.yingjia@163.com School of Mathematics and Statistics, Beihua University, Jilin, 132013, China

\section{Springer}

\begin{abstract}
We formulate a stochastic SIS epidemic model with vaccination by introducing a Lévy noise and regime switching into the epidemic model. First, we prove that the stochastic model admits a unique global positive solution. Moreover, we study the asymptotic behavior of the stochastic regime switching SIS model with vaccination driven by Lévy noise.
\end{abstract}

Keywords: stochastic SIS epidemic model; vaccination; Lévy noise; Markov switching; global positive solution; asymptotic behavior

\section{Introduction}

It is well known that epidemic diseases (tuberculosis, SARS, AIDS, etc.) always bring great harm to the stability of the social and threat individual health, Moreover, the rapid development of society and frequent contacts among people can accelerate the spread of epidemic disease. In recent years, control of infectious diseases becomes an increasingly complex issue. Vaccination is an important strategy for the elimination of infectious disease. Communication diseases may be prevented by a valid vaccination [1-3]. The vaccination enables the vaccinated to get a permanent or temporary immunity. When the immunity is temporary, it can be lost after a period of time. It is often assumed that the process of losing immunity is exponential [4-6].

To formulate epidemic models with vaccination, we often let $S(t)$ to denote the number of members that are susceptible to infections at time $t, I(t)$ denotes the number of members that infective at time $t$, and $V(t)$ denotes the number of members that are immune to infections at time $t$ as the result of vaccination. The total population size at time $t$ is equal to $S+I+V$ for an SIS model with vaccination. The deterministic SIS epidemic model with vaccination is expressed by

$$
\left\{\begin{array}{l}
\dot{S}(t)=(1-q) A-\beta I(t) S(t)-(\mu+p) S(t)+\gamma I(t)+\varepsilon V(t), \\
\dot{I}(t)=\beta S(t) I(t)-(\mu+\gamma+\alpha) I(t), \\
\dot{V}(t)=q A+p S(t)-(\mu+\varepsilon) V(t),
\end{array}\right.
$$

where $A$ is a constant input of new members into the population per unit time, $q$ ( $0 \leq$ $q \leq 1)$ is the fraction of vaccinated for newborns; $\beta$ represents the transmission coefficient

(c) The Author(s) 2017. This article is distributed under the terms of the Creative Commons Attribution 4.0 International License (http://creativecommons.org/licenses/by/4.0/), which permits unrestricted use, distribution, and reproduction in any medium, provided you give appropriate credit to the original author(s) and the source, provide a link to the Creative Commons license, and indicate if changes were made. 
between compartments $S$ and $I, \mu$ is the natural death rate per capita, $p$ represents the proportional coefficient of vaccinated for the susceptible, $\gamma$ is the recovery rate, $\varepsilon$ is the rate of losing immunity for vaccinated individuals, and $\alpha$ represents the disease-caused death rate of infectious individuals. These parameter values are all nonnegative, and $\mu, A>0$. Li and Ma [6] analyzed the thresholds, equilibria, and stabilities of the epidemic model (1.1) of SIS type with vaccination. For system (1.1), there exists the basic reproduction number $R_{0}$. The asymptotic behavior is globally asymptotically stable convergence to a disease-free equilibrium $P_{0}\left(S_{0}, I_{0}, V_{0}\right)$ below the threshold $R_{0}$. Otherwise, $P_{0}$ is unstable when $R_{0}>1$, and there is an endemic equilibrium $P^{*}\left(S^{*}, I^{*}, V^{*}\right)$, which is globally asymptotically stable.

Because of full randomness and stochasticity in real life, many studies have indicated that environmental fluctuations have a huge impact on the transmission of an epidemic $[7,8]$. Zhao and Jiang [9] considered the following stochastically perturbed SIS epidemic model with vaccination:

$$
\left\{\begin{aligned}
d S(t)= & {[(1-q) A-\beta I(t) S(t)-(\mu+p) S(t)+\gamma I(t)+\varepsilon V(t)] d t } \\
& +\sigma_{1} S(t) d B_{1}(t) \\
d I(t)= & {[\beta S(t)-(\mu+\gamma+\alpha)] I(t) d t+\sigma_{2} I(t) d B_{2}(t) } \\
d V(t)= & {[q A+p S(t)-(\mu+\varepsilon) V(t)] d t+\sigma_{3} V(t) d B_{3}(t) }
\end{aligned}\right.
$$

where $B_{i}(t)(i=1,2,3)$ are independent Brownian motions, and $\sigma_{i}(i=1,2,3)$ are their intensities. They showed that when the perturbations and the disease-related death rate $\alpha$ are small, there is a stationary distribution, and it is ergodic as the reproductive number of the deterministic model $R_{0}>1$. If $R_{0} \leq 1$, then the solution of model (1.2) is oscillating around the disease-free equilibrium $P_{0}$.

Furthermore, the population may suffer sudden environmental shocks and catastrophes such as climate charges (earthquakes, hurricanes, etc.) and unpredictable disasters. These phenomena cannot be modeled by stochastic continuous models. Bao et al. suggested that the non-Gaussian Lévy noise should be suitable for describing these phenomena [10-14]. They considered stochastic Lotka-Volterra population systems with jumps [15] for the first time, and then some important results that reveal that jump processes can bring their effect on the properties of the systems have been reported [16-18]. There are also many results on the epidemic models with jumps [19-22]. Chen and Kang [23] introduced a Lévy noise into the multistrain SIS epidemic model and investigated its effects on the spread of infectious disease with multiple pathogen strains.

However, epidemic models may be perturbed by colored noise, which can cause the system switching from one environmental regime to another [14]. For example, the transmission rate in winter will be much different from that in summer. Often, the switching between environmental regimes is often memoryless, and the waiting time for the next switching follows the exponential distribution [24]. Thus, we use a continue-time Markov chain $r(t)$ to model random environments with colored noise. Let $r(t)(t \geq 0)$ be a rightcontinuous Markov chain on the probability space $(\Omega, \mathfrak{F}, P)$ taking values in a finite state space $\mathbb{M}=\{1,2, \ldots, N\}$ with generator $\Gamma=\left(\gamma_{i j}\right)_{N \times N}$, that is,

$$
P\{r(t+\delta)=j \mid r(t)=i\}= \begin{cases}\gamma_{i j} \delta+o(\delta) & \text { if } i \neq j, \\ 1+\gamma_{i j} \delta+o(\delta) & \text { if } i=j,\end{cases}
$$


where $\gamma_{i j} \geq 0$ is the transition rate from $i$ to $j$ with $i \neq j$ whereas $\gamma_{i i}=-\sum_{j \neq i} \gamma_{i j}$. We assume that the Markov chain and Brownian motion are independent.

In this paper, we set up a stochastic regime switching SIS model with vaccination driven by a Lévy noise:

$$
\left\{\begin{aligned}
d S(t)= & {[(1-q(r(t))) A(r(t))-\beta(r(t)) I(t) S(t)} \\
& -(\mu(r(t))+p(r(t))) S(t)+\gamma(r(t)) I(t)+\varepsilon(r(t)) V(t)] d t \\
& +\sigma_{1}(r(t)) S(t) d B_{1}(t)+\int_{U} D_{1}(r(t), u) S(t) \widetilde{N}(d t, d u), \\
d I(t)= & {[\beta(r(t)) S(t)-(\mu(r(t))+\gamma(r(t))+\alpha(r(t)))] I(t) d t } \\
& +\sigma_{2}(r(t)) I(t) d B_{2}(t)+\int_{U} D_{2}(r(t), u) I(t) \widetilde{N}(d t, d u), \\
d V(t)= & {[q(r(t)) A(r(t))+p(r(t)) S(t)-(\mu(r(t))+\varepsilon(r(t))) V(t)] d t } \\
& +\sigma_{3}(r(t)) V(t) d B_{3}(t)+\int_{U} D_{3}(r(t), u) V(t) \widetilde{N}(d t, d u),
\end{aligned}\right.
$$

where $D_{i}(r(t), u)>-1(i=1,2,3), \tilde{N}(d t, d u)$ is the compensated Poisson random measure given by $\tilde{N}(d t, d u)=N(d t, d u)-v(d u) d t$, and $v$ is the characteristic measure of $N$ on a measurable subset $U$ of $[0, \infty)$ satisfying $v(d u)<\infty$. Since stochastic model (1.3) is perturbed by both Lévy noise and colored noise, its dynamics is an interesting and important question.

The paper is organized as follows. In Section 2, we give some notation and the equivalent form of the studied model. In Section 3, we study the global positive solution of model (1.3). In Sections 4 and 5, we investigate the asymptotic behavior of the stochastic regime switching SIS model with vaccination driven by a Lévy noise.

\section{Preliminaries}

Here we assume that the Brownian motion and Markov chain are independent. In this paper, we assume that $\gamma_{i j}>0$ for $i \neq j$, and $q(k), A(k), \beta(k), \mu(k), p(k), \gamma(k), \varepsilon(k)$, and $\alpha(k)$ are all positive constants for each $k \in \mathbb{M}$. System (1.3) can be regarded as the result of a stochastic SIS model with vaccination

$$
\left\{\begin{aligned}
d S(t)= & {[(1-q(k)) A(k)-\beta(k) I(t) S(t)-(\mu(k)+p(k)) S(t)} \\
& +\gamma(k) I(t)+\varepsilon(k) V(t)] d t \\
& +\sigma_{1}(k) S(t) d B_{1}(t)+\int_{U} D_{1}(k, u) S(t) \widetilde{N}(d t, d u), \\
d I(t)= & {[\beta(k) S(t)-(\mu(k)+\gamma(k)+\alpha(k))] I(t) d t+\sigma_{2}(k) I(t) d B_{2}(t) } \\
& +\int_{U} D_{2}(k, u) I(t) \widetilde{N}(d t, d u), \\
d V(t)= & {[q(k) A(k)+p(k) S(t)-(\mu(k)+\varepsilon(k)) V(t)] d t+\sigma_{3}(k) V(t) d B_{3}(t) } \\
& +\int_{U} D_{3}(k, u) V(t) \widetilde{N}(d t, d u),
\end{aligned}\right.
$$

switching from one to the others according to the movement of the Markov chain. For the corresponding regime switching SIS model with vaccination of system (2.1), there exists the disease-free equilibrium $P_{k}^{0}\left(S_{k}^{0}, I_{k}^{0}, V_{k}^{0}\right)=\left(\frac{A(k)}{\mu(k)} \frac{\mu(k)(1-q(k))+\varepsilon(k)}{\mu(k)+\varepsilon(k)+p(k)}, 0, \frac{A(k)}{\mu(k)} \frac{\mu(k) q(k)+p(k)}{\mu(k)+\varepsilon(k)+p(k)}\right)$ when the threshold $R_{k}^{0} \leq 1$ for $k \in \mathbb{M}$. Otherwise, there exists an endemic equilibrium $P_{k}^{*}\left(S_{k}^{*}, I_{k}^{*}, V_{k}^{*}\right)$ such that

$$
(1-q(k)) A(k)=\beta(k) I_{k}^{*} S_{k}^{*}+(\mu(k)+p(k)) S_{k}^{*}-\gamma(k) I_{k}^{*}-\varepsilon(k) V_{k}^{*}
$$


and

$$
\beta(k) S_{k}^{*} I_{k}^{*}=(\mu(k)+\gamma(k)+\alpha(k)) I_{k}^{*}, \quad q(k) A(k)+p(k) S_{k}^{*}=(\mu(k)+\varepsilon(k)) V_{k}^{*}
$$

for $k \in \mathbb{M}$.

\section{Existence and uniqueness of positive solution}

Our first concern is whether the solution has a global existence. Moreover, we also consider whether, as a population dynamic model, the value is nonnegative. Therefore, we guarantee the existence of a global positive solution under some assumptions.

For the jump diffusion coefficient, we assume that, for each $m>0$, there exists $L_{m}>0$ such that

(H1) $\int_{U}\left|H_{i}(x, u, k)-H_{j}(y, u, k)\right|^{2} v(d u) \leq L_{m}|x-y|^{2}(i=1,2,3, k \in \mathbb{M})$, where $H_{1}(x, u, k)=D_{1}(k, u) S(t), H_{2}(x, u, k)=D_{2}(k, u) I(t), H_{3}(x, u, k)=D_{3}(k, u) V(t)$ with $|x| \vee|y| \leq m$;

(H2) $\left|\log \left(1+D_{i}(k, u)\right)\right|<\infty$ for $D_{i}(k, u)>-1(i=1,2,3, k \in \mathbb{M})$.

Theorem 3.1 Let assumptions (H1) and (H2) hold. Then, for any given initial value $(S(0), I(0), V(0)) \in R_{+}^{3}$, there is a unique solution $(S(t), I(t), V(t))$ of system (1.3) on $t \geq 0$ almost surely, and the solution remains in $R_{+}^{3}$ with probability 1.

Proof Since the drift and the diffusion of system (1.3) are both locally Lipschitz, for any given initial value $(S(0), I(0), V(0)) \in R_{+}^{3}$, there is a unique local solution $(S(t), I(t), V(t)) \in$ $R_{+}^{3}$ for any $t \in\left[0, \tau_{e}\right)$, where $\tau_{e}$ is the explosion time [25]. Let $\eta_{0}>0$ be sufficiently large such that

$$
\tau_{\eta}=\inf \left\{t \in\left[0, \tau_{e}\right): S(t) \notin\left(\frac{1}{\eta}, \eta\right), I(t) \notin\left(\frac{1}{\eta}, \eta\right) \text {, or } V(t) \notin\left(\frac{1}{\eta}, \eta\right)\right\} \text {. }
$$

Obviously, $\tau_{\eta}$ is increasing as $\eta \rightarrow \infty$, and $\tau_{\infty}=\lim _{\eta \rightarrow \infty} \tau_{\eta} \leq \tau_{e}$ a.s. To show that the solution is global, it suffices to show that $\tau_{\infty}=\infty$ a.s.

Consider the following Lyapunov function:

$$
W(S, I, V, k)=c_{1}(k)(S-1-\log S)+c_{2}(k)(I-1-\log I)+c_{3}(k)(V-1-\log V),
$$

where $c_{i}(k)(i=1,2,3)$ are positive constants for all $k \in \mathbb{M}$.

Set $T>0$ be arbitrary. Then, for any $0<t<\tau_{\eta} \wedge T$, we have

$$
\begin{aligned}
d W(S, I, V, k) \\
=L W(S, I, V, k) d t+c_{1}(k) \sigma_{1}(k)(S-1) d B_{1}(t)+c_{2}(k) \sigma_{2}(k)(I-1) d B_{2}(t) \\
\quad+c_{3}(k) \sigma_{3}(k)(V-1) d B_{3}(t)+\int_{U}\left[c_{1}(k)\left(D_{1}(k, u) S-\log \left(1+D_{1}(k, u)\right)\right)\right. \\
\quad+c_{2}(k)\left(D_{2}(k, u) I-\log \left(1+D_{2}(k, u)\right)\right)+c_{3}(k)\left(D_{3}(k, u) V\right. \\
\left.\left.\quad-\log \left(1+D_{3}(k, u)\right)\right)\right] \tilde{N}(d t, d u),
\end{aligned}
$$


where

$L W(S, I, V, k)$

$$
\begin{aligned}
\leq & c_{1}(k)(1-q(k)) A(k)+c_{1}(k)(\mu(k)+p(k))+c_{2}(k)(\mu(k)+\gamma(k)+\alpha(k)) \\
& +c_{3}(k)(q(k) A(k)+\mu(k)+\varepsilon(k))-\left(c_{1}(k)-c_{2}(k)\right) \beta(k) S I-\left(c_{1}(k)(\mu(k)\right. \\
& \left.+p(k))+c_{2}(k) \beta(k)-c_{3}(k) p(k)\right) S-\left(c_{2}(k)(\mu(k)+\gamma(k)+\alpha(k))-c_{1}(k)\right. \\
& \times(\gamma(k)+\beta(k))) I-\left(c_{3}(k)(\mu(k)+\varepsilon(k))-c_{1}(k) \varepsilon(k)\right) V-c_{1}(k)(1-q(k)) A(k) \frac{1}{S} \\
& -c_{1}(k) \gamma(k) \frac{I}{S}-c_{1}(k) \varepsilon(k) \frac{V}{S}-c_{3}(k) q(k) A(k) \frac{1}{V}-c_{3}(k) p(k) \frac{S}{V}+\frac{1}{2} c_{1}(k) \sigma_{1}^{2}(k) \\
& +\frac{1}{2} c_{2}(k) \sigma_{2}^{2}(k)+\frac{1}{2} c_{3}(k) \sigma_{3}^{2}(k)+\int_{U}\left[c_{1}(k)\left(D_{1}(k, u)-\log \left(1+D_{1}(k, u)\right)\right)\right. \\
& \left.+c_{2}(k)\left(D_{2}(k, u)-\log \left(1+D_{2}(k, u)\right)\right)+c_{3}(k)\left(D_{3}(k, u)-\log \left(1+D_{3}(k, u)\right)\right)\right] v(d u) \\
& +\sum_{l=1}^{N} \gamma_{k l} W(S, I, V, l) .
\end{aligned}
$$

Choose

$$
\begin{aligned}
& c_{1}(k)>c_{2}(k), \quad c_{1}(k)(\mu(k)+p(k))+c_{2}(k) \beta(k)>c_{3}(k) p(k), \\
& c_{3}(k)(\mu(k)+\varepsilon(k))>c_{1}(k) \varepsilon(k), \quad \text { and } \\
& c_{2}(k)(\mu(k)+\gamma(k)+\alpha(k))>c_{1}(k)(\gamma(k)+\beta(k)) \quad \text { for } k \in \mathbb{M} .
\end{aligned}
$$

By Assumption (H2) and the inequality $D_{i}(k, u)-\log \left(1+D_{i}(k, u)\right) \geq 0$ for $D_{i}(k, u)>-1$, we have

$$
\begin{aligned}
L W \leq & c_{1}(k)(1-q(k)) A(k)+c_{1}(k)(\mu(k)+p(k))+c_{2}(k)(\mu(k)+\gamma(k)+\alpha(k)) \\
& +c_{3}(k)(q(k) A(k)+\mu(k)+\varepsilon(k))+\frac{1}{2} c_{1}(k) \sigma_{1}^{2}(k)+\frac{1}{2} c_{2}(k) \sigma_{2}^{2}(k) \\
& +\frac{1}{2} c_{3}(k) \sigma_{3}^{2}(k)+3 K+\sum_{l=1}^{N} \gamma_{k l} V(S, I, V, l),
\end{aligned}
$$

where $K=\max _{1 \leq i \leq 3}\left\{\int_{U} c_{i}(k)\left(D_{i}(k, u)-\log \left(1+D_{i}(k, u)\right)\right) v(d u)\right\}$.

Let $\check{c}=\max \left\{\frac{c_{i}(l)}{c_{i}(k)}: 1 \leq i \leq 3,1 \leq l, k \leq N\right\}$. Then, for any $l, k \in \mathbb{M}$, we get

$$
\begin{aligned}
W(S, I, V, l) & \leq \check{c}\left[c_{1}(k)(S-1-\log S)+c_{2}(k)(I-1-\log I)+c_{3}(k)(V-1-\log V)\right] \\
& =\check{c} W(S, I, V, k) .
\end{aligned}
$$

Therefore

$$
\sum_{l=1}^{N} \gamma_{k l} W(S, I, V, l) \leq \check{c}\left(\sum_{l=1}^{N}\left|\gamma_{k l}\right|\right) W(S, I, V, k),
$$


and thus

$$
\begin{aligned}
L W(S, I, V, k) & :=\bar{K}+\sum_{l=1}^{N} \gamma_{k l} W(S, I, V, k) \\
& \leq \tilde{K}[1+W(S, I, V, k)],
\end{aligned}
$$

where

$$
\begin{aligned}
\tilde{K}= & \max \left\{\bar{K}, \check{c}\left(\sum_{l=1}^{N}\left|\gamma_{k l}\right|\right)\right\}, \\
\bar{K}= & c_{1}(k)(1-q(k)) A(k)+c_{1}(k)(\mu(k)+p(k))+c_{2}(k)(\mu(k)+\gamma(k)+\alpha(k)) \\
& +c_{3}(k)(q(k) A(k)+\mu(k)+\varepsilon(k))+\frac{1}{2} c_{1}(k) \sigma_{1}^{2}(k)+\frac{1}{2} c_{2}(k) \sigma_{2}^{2}(k)+\frac{1}{2} c_{3}(k) \sigma_{3}^{2}(k)+3 K .
\end{aligned}
$$

Integrating both sides of (3.2) from 0 to $\tau_{\eta} \wedge T$ and taking expectation yield

$$
\begin{aligned}
& E W\left(S\left(\tau_{\eta} \wedge T\right), I\left(\tau_{\eta} \wedge T\right), V\left(\tau_{\eta} \wedge T\right), r\left(\tau_{\eta} \wedge T\right)\right) \\
& \quad \leq W(S(0), I(0), V(0), r(0))+E \int_{0}^{\tau_{\eta} \wedge T} L W(S(\tau), I(\tau), V(\tau), r(\tau)) d \tau \\
& \quad \leq[W(S(0), I(0), V(0), r(0))+\tilde{K} T] e^{\tilde{K} T} .
\end{aligned}
$$

In fact, we find that

$$
\begin{aligned}
& {[W(S(0), I(0), V(0), r(0))+\tilde{K} T] e^{\tilde{K} T}} \\
& \quad \geq E\left[1_{\left\{\tau_{\eta} \wedge T\right\}} W\left(S\left(\tau_{m}, \omega\right), I\left(\tau_{m}, \omega\right), V\left(\tau_{m}, \omega\right), r\left(\tau_{m}, \omega\right)\right)\right] \\
& \quad \geq \min _{1 \leq i \leq 3}\left\{c_{i}(k)(\eta-1-\log \eta), c_{i}(k)\left(\frac{1}{\eta}-1+\log \eta\right)\right\} P\left(\tau_{\eta} \leq T\right),
\end{aligned}
$$

where $1_{\left\{\tau_{\eta} \wedge T\right\}}$ is the indicator function of $\left\{\tau_{\eta} \wedge T\right\}$. Letting $\eta \rightarrow \infty$ implies

$$
P\left(\tau_{\infty} \leq T\right)=0 .
$$

By the arbitrariness of $T$ we can see that

$$
P\left(\tau_{\infty}=\infty\right)=1 .
$$

Thus, the proof is complete.

\section{Asymptotic behavior around $P_{\boldsymbol{k}}^{0}$}

It is clear that $P_{k}^{0}\left(S_{k}^{0}, 0, V_{k}^{0}\right)$ is the solution for the corresponding regime switching SIS model with vaccination of system (1.3), which is called the disease-free equilibrium. If $R_{k}^{0} \leq 1$, then $P_{k}^{0}$ is globally asymptotically stable. This means that the disease will disappear after some period of time. Whereas for the stochastic regime switching SIS model with vaccination driven by a Lévy noise, $P_{k}^{0}$ is no longer a disease-free equilibrium. Thus for the solution of system (1.3), what kind of changes will appear around $P_{k}^{0}$ ? In this section, we study the asymptotic behavior around $P_{k}^{0}$. 
Theorem 4.1 Let $(S(t), I(t), V(t))$ be the solution of system (1.3) with any initial value $(S(0), I(0), V(0)) \in R_{+}^{3}$. Suppose that $R_{k}^{0} \leq 1$ and the following conditions are satisfied:

$$
\begin{aligned}
& \mu(k)\left(\frac{2(\mu(k)+p(k))}{\varepsilon(k)}+1\right)>\left(1+a_{2}(k)\right) \sigma_{1}^{2}(k)+\left(3+a_{2}(k)\right) \int_{U} D_{1}^{2}(k, u) v(d u), \\
& (\mu(k)+\alpha(k))\left(1+a_{2}(k)\right)>\frac{1}{2} \sigma_{2}^{2}(k)\left(1+a_{2}(k)\right)+\left(3+a_{2}(k)\right) \int_{U} D_{2}^{2}(k, u) v(d u),
\end{aligned}
$$

and

$$
\mu(k)>\sigma_{3}^{2}(k)+3 \int_{U} D_{3}^{2}(k, u) v(d u) .
$$

Then

$$
\begin{gathered}
\limsup _{t \rightarrow \infty} \frac{1}{t} E \int_{0}^{t}\left[\left(S(s)-S_{k}^{0}\right)+I^{2}(s)+\left(V(s)-V_{k}^{0}\right)^{2}\right] d s \\
\leq \frac{1}{\tilde{M}}\left[\sigma_{1}^{2}(k)\left(S_{k}^{0}\right)^{2}\left(1+a_{2}(k)\right)+\sigma_{3}^{2}(k)\left(V_{k}^{0}\right)^{2}\right],
\end{gathered}
$$

where

$$
\begin{aligned}
a_{2}(k)= & \frac{2 \mu(k)}{\varepsilon(k)} \\
\tilde{M}= & \min \left\{\mu(k)\left(\frac{2(\mu(k)+p(k))}{\varepsilon(k)}+1\right)-\left(1+a_{2}(k)\right) \sigma_{1}^{2}(k)-\left(3+a_{2}(k)\right) \int_{U} D_{1}^{2}(k, u) v(d u),\right. \\
& (\mu(k)+\alpha(k))\left(1+a_{2}(k)\right)-\frac{1}{2} \sigma_{2}^{2}(k)\left(1+a_{2}(k)\right)-\left(3+a_{2}(k)\right) \int_{U} D_{2}^{2}(k, u) v(d u), \\
& \left.\mu(k)-\sigma_{3}^{2}(k)-3 \int_{U} D_{3}^{2}(k, u) v(d u)\right\}
\end{aligned}
$$

Proof First, by the change of variables

$$
x(t)=S(t)-S_{k}^{0}, \quad y(t)=I(t), \quad z(t)=V(t)-V_{k}^{0}
$$

system (2.1) can be rewritten as follows:

$$
\left\{\begin{aligned}
d x(t)= & {\left[-\beta(k)\left(x(t)+S_{k}^{0}\right) y(t)-(\mu(k)+p(k)) x(t)+\gamma(k) y(t)+\varepsilon(k) z(t)\right] d t } \\
& +\sigma_{1}(k)\left(x(t)+S_{k}^{0}\right) d B_{1}(t)+\int_{U} D_{1}(k, u)\left(x(t)+S_{k}^{0}\right) \widetilde{N}(d t, d u), \\
d y(t)= & {\left[\beta(k) x(t) y(t)+\left(\beta(k) S_{k}^{0}-(\mu(k)+\gamma(k)+\alpha(k))\right) y(t)\right] d t } \\
& +\sigma_{2}(k) y(t) d B_{2}(t)+\int_{U} D_{2}(k, u) y(t) \widetilde{N}(d t, d u), \\
d z(t)= & {[p(k) x(t)-(\mu(k)+\varepsilon(k)) z(t)] d t+\sigma_{3}(k)\left(z(t)+V_{k}^{0}\right) d B_{3}(t) } \\
& +\int_{U} D_{3}(k, u)\left(z(t)+V_{k}^{0}\right) \widetilde{N}(d t, d u) .
\end{aligned}\right.
$$

Consider the $C^{2}$ function

$$
W(x, y, z, k)=a_{1}(k) y+\frac{a_{2}(k)}{2}(x+y)^{2}+\frac{1}{2}(x+y+z)^{2},
$$


where $k \in \mathbb{M}, a_{1}(k), a_{2}(k)$ are positive constants to be determined later. Using Itô's formula, we obtain

$$
\begin{aligned}
d W= & L W d t+\sigma_{1}(k)\left(x+S_{k}^{0}\right)\left[a_{2}(k)(x+y)+x+y+z\right] d B_{1}(t) \\
& +\sigma_{2}(k) y\left[a_{1}(k)+a_{2}(k)(x+y)+x+y+z\right] d B_{2}(t) \\
& +\sigma_{3}(k)(x+y+z)\left(z+V_{k}^{2}\right) d B_{3}(t)+a_{1}(k) \int_{U} D_{2}(k, u) y \widetilde{N}(d t, d u) \\
& +\frac{a_{2}(k)}{2} \int_{U}\left[\left(D_{1}(k, u) x+D_{2}(k, u) y\right)^{2}\right. \\
& \left.+2(x+y)\left(D_{1}(k, u) x+D_{2}(k, u) y\right)\right] \tilde{N}(d t, d u) \\
& +\int_{U}\left[(x+y+z)\left(D_{1}(k, u) x+D_{2}(k, u) y+D_{3}(k, u) z\right)\right. \\
& \left.+\left(D_{1}(k, u) x+D_{2}(k, u) y+D_{3}(k, u) z\right)^{2}\right] \tilde{N}(d t, d u),
\end{aligned}
$$

where

$$
\begin{aligned}
L W= & -\left[a_{2}(k)(\mu(k)+p(k))+\mu(k)\right] x^{2}-\left(a_{2}(k)+1\right)(\mu(k)+\alpha(k)) y^{2} \\
& -\mu(k) z^{2}+a_{1}(k)\left[\beta(k) S_{k}^{0}\right. \\
& -(\mu(k)+\alpha(k)+\gamma(k))] y+\left[a_{1}(k) \beta(k)-a_{2}(k)(2 \mu(k)+\alpha(k)\right. \\
& +p(k))-(2 \mu(k)+\alpha(k))] x y \\
& +\left[a_{2}(k) \varepsilon(k)-(2 \mu(k)+\alpha(k))\right] y z+\left(a_{2}(k) \varepsilon(k)-2 \mu(k)\right) x z \\
& +\frac{1}{2} \sigma_{1}^{2}(k)\left(a_{2}(k)+1\right)\left(x+S_{k}^{0}\right)^{2} \\
& +\frac{1}{2} \sigma_{2}^{2}(k)\left(a_{2}(k)+1\right) y^{2}+\frac{1}{2} \sigma_{3}^{2}(k)\left(z+V_{k}^{0}\right)^{2} \\
& +\frac{a_{2}(k)}{2} \int_{U}\left[\left(D_{1}(k, u) x+D_{2}(k, u) y\right]^{2} v(d u)\right. \\
& +\int_{U}\left[\left(D_{1}(k, u) x+D_{2}(k, u) y+D_{3}(k, u) z\right]^{2} v(d u)+\sum_{l=1}^{N} \gamma_{k l} W(x, y, z, l) .\right.
\end{aligned}
$$

Note that

$$
\begin{aligned}
& a_{1}(k) \beta(k)-a_{2}(k)(2 \mu(k)+\alpha(k)+p(k))-(2 \mu(k)+\alpha(k))=0, \\
& a_{2}(k) \varepsilon(k)-2 \mu(k)=0 .
\end{aligned}
$$

Applying the inequalities $2 a b \leq a^{2}+b^{2},(a+b)^{2} \leq 2 a^{2}+2 b^{2}$, and $(a+b+c)^{3} \leq 3 a^{2}+3 b^{2}+$ $3 c^{2}$, we get

$$
\begin{aligned}
L W \leq & -\left[\mu(k)\left(\frac{2(\mu(k)+p(k))}{\varepsilon(k)}+1\right)-\left(1+\frac{2 \mu(k)}{\varepsilon(k)}\right) \sigma_{1}^{2}(k)\right. \\
& \left.-\left(\frac{2 \mu(k)}{\varepsilon(k)}+3\right) \int_{U} D_{1}^{2}(k, u) v(d u)\right] x^{2}
\end{aligned}
$$




$$
\begin{aligned}
& -\left[\left(1+\frac{2 \mu(k)}{\varepsilon(k)}\right)(\mu(k)+\alpha(k))-\frac{1}{2} \sigma_{2}^{2}(k)\left(1+\frac{2 \mu(k)}{\varepsilon(k)}\right)\right. \\
& \left.-\left(\frac{2 \mu(k)}{\varepsilon(k)}+3\right) \int_{U} D_{2}^{2}(k, u) v(d u)\right] y^{2} \\
& -\left[\mu(k)-\sigma_{3}^{2}(k)-3 \int_{U} D_{3}^{2}(k, u) v(d u)\right] z^{2}+\sigma_{3}^{2}(k)\left(V_{k}^{0}\right)^{2} \\
& +\sigma_{1}^{2}(k)\left(1+\frac{2 \mu(k)}{\varepsilon(k)}\right)\left(S_{k}^{0}\right)^{2} \\
& +\sum_{l=1}^{N} \gamma_{k l} W(x, y, z, l) .
\end{aligned}
$$

Denote $\check{a}=\max \left\{\frac{a_{i}(l)}{a_{i}(k)}, 1 \leq i \leq 2,1 \leq l, k \leq N\right\}$. Then

$$
\begin{aligned}
\sum_{l=1}^{N} \gamma_{k l} W(x, y, z, l) & \leq \check{a}\left(\sum_{l=1}^{N}\left|\gamma_{k l}\right|\right) W(x, y, z, k) \\
& :=M_{1} W(x, y, z, k),
\end{aligned}
$$

and thus

$$
\begin{aligned}
L W \leq & -\left[\mu(k)\left(\frac{2(\mu(k)+p(k))}{\varepsilon(k)}+1\right)-\left(1+\frac{2 \mu(k)}{\varepsilon(k)}\right) \sigma_{1}^{2}(k)\right. \\
& \left.-\left(\frac{2 \mu(k)}{\varepsilon(k)}+3\right) \int_{U} D_{1}^{2}(k, u) v(d u)\right] x^{2} \\
& -\left[\left(1+\frac{2 \mu(k)}{\varepsilon(k)}\right)(\mu(k)+\alpha(k))-\frac{1}{2} \sigma_{2}^{2}(k)\left(1+\frac{2 \mu(k)}{\varepsilon(k)}\right)\right. \\
& \left.-\left(\frac{2 \mu(k)}{\varepsilon(k)}+3\right) \int_{U} D_{2}^{2}(k, u) v(d u)\right] y^{2} \\
& -\left[\mu(k)-\sigma_{3}^{2}(k)-3 \int_{U} D_{3}^{2}(k, u) v(d u)\right] z^{2}+\sigma_{3}^{2}(k)\left(V_{k}^{0}\right)^{2} \\
& +\sigma_{1}^{2}(k)\left(1+\frac{2 \mu(k)}{\varepsilon(k)}\right)\left(S_{k}^{0}\right)^{2} \\
& +M_{1} W(x, y, z, k) .
\end{aligned}
$$

Integrating both sides of (4.2) from 0 to $t$ and taking expectation, we obtain

$$
\begin{aligned}
0 \leq & E W(x(t), y(t), z(t), r(t)) \\
= & W(x(0), y(0), z(0), r(0))+E \int_{0}^{t} L W d s \\
\leq & W(x(0), y(0), z(0), r(0))-E \int_{0}^{t}\left\{\left[\mu(k)\left(\frac{2(\mu(k)+p(k))}{\varepsilon(k)}+1\right)-\left(1+\frac{2 \mu(k)}{\varepsilon(k)}\right) \sigma_{1}^{2}(k)\right.\right. \\
& \left.-\left(\frac{2 \mu(k)}{\varepsilon(k)}+3\right) \int_{U} D_{1}^{2}(k, u) v(d u)\right] x^{2}(s) \\
& +\left[\left(1+\frac{2 \mu(k)}{\varepsilon(k)}\right)(\mu(k)+\alpha(k))-\frac{1}{2} \sigma_{2}^{2}(k)\left(1+\frac{2 \mu(k)}{\varepsilon(k)}\right)\right.
\end{aligned}
$$




$$
\begin{aligned}
& \left.\left.-\left(\frac{2 \mu(k)}{\varepsilon(k)}+3\right) \int_{U} D_{2}^{2}(k, u) v(d u)\right] y^{2}+\left[\mu(k)-\sigma_{3}^{2}(k)-3 \int_{U} D_{3}^{2}(k, u) v(d u)\right] z^{2}\right\} d s \\
& +\left[\sigma_{3}^{2}(k)\left(V_{k}^{0}\right)^{2}+\sigma_{1}^{2}(k)\left(1+\frac{2 \mu(k)}{\varepsilon(k)}\right)\left(S_{k}^{0}\right)^{2}\right] t+M_{1} E \int_{0}^{t} W(x(s), y(s), z(s), r(s)) d s \\
\leq & \{W(x(0), y(0), z(0), r(0)) \\
& -E \int_{0}^{t}\left\{\left[\mu(k)\left(\frac{2(\mu(k)+p(k))}{\varepsilon(k)}+1\right)-\left(1+\frac{2 \mu(k)}{\varepsilon(k)}\right) \sigma_{1}^{2}(k)\right.\right. \\
& \left.-\left(\frac{2 \mu(k)}{\varepsilon(k)}+3\right) \int_{U} D_{1}^{2}(k, u) v(d u)\right] x^{2}(s) \\
& +\left[\left(1+\frac{2 \mu(k)}{\varepsilon(k)}\right)(\mu(k)+\alpha(k))-\frac{1}{2} \sigma_{2}^{2}(k)\left(1+\frac{2 \mu(k)}{\varepsilon(k)}\right)\right. \\
& \left.\left.-\left(\frac{2 \mu(k)}{\varepsilon(k)}+3\right) \int_{U} D_{2}^{2}(k, u) v(d u)\right] y^{2}+\left[\mu(k)-\sigma_{3}^{2}(k)-3 \int_{U} D_{3}^{2}(k, u) v(d u)\right] z^{2}\right\} d s \\
& \left.+\left[\sigma_{3}^{2}(k)\left(V_{k}^{0}\right)^{2}+\sigma_{1}^{2}(k)\left(1+\frac{2 \mu(k)}{\varepsilon(k)}\right)\left(S_{k}^{0}\right)^{2}\right] t\right\} e^{M_{1} t},
\end{aligned}
$$

which implies that

$$
\begin{gathered}
\limsup _{t \rightarrow \infty} \frac{1}{t} E \int_{0}^{t}\left[\left(S(s)-S_{k}^{0}\right)^{2}+I^{2}(s)+\left(V(s)-V_{k}^{0}\right)^{2}\right] d s \\
\leq \frac{1}{\tilde{M}}\left[\sigma_{3}^{2}(k)\left(V_{k}^{0}\right)^{2}+\sigma_{1}^{2}(k)\left(1+\frac{2 \mu(k)}{\varepsilon(k)}\right)\left(S_{k}^{0}\right)^{2}\right],
\end{gathered}
$$

where $\tilde{M}$ are defined as in Theorem 4.1. This completes the proof.

Remark 4.1 From Theorem 4.1 we can see that the solution of system (1.3) will oscillate around the disease-free equilibrium $P_{k}^{0}\left(S_{k}^{0}, 0, V_{k}^{0}\right)$ under some conditions. The lower the vibration intensity of the Lévy noise, the nearer the solution of the stochastic SIS model (1.3) to the disease-free equilibrium $P_{k}^{0}$. Hence the disease will die out. Besides, if $D_{i}(k, u)=$ $0(i=1,2,3)$, then Theorem 4.1 shows the asymptotic behavior of the stochastic regime switching SIS model with vaccination. Then the solution will oscillate around the diseasefree equilibrium where the intensity is relevant to the values of $\sigma_{i}(k)(i=1,2,3)$.

\section{Asymptotic behavior around $P_{k}^{*}$}

In this section, we assume that $R_{0}^{k}>1$. Then $P_{k}^{*}$ is the endemic equilibrium of the corresponding regime switching SIS model with vaccination for system (1.3). But it is no longer an endemic equilibrium of system (1.3). Similarly, we also expect to find out whether or not the solution goes around $P_{k}^{*}$. We get the following result.

Theorem 5.1 Let $(S(t), I(t), V(t))$ be the solution of system (1.3) with any initial value $(S(0), I(0), V(0)) \in R_{+}^{3}$. Suppose that $R_{k}^{0}>1$ and the following conditions are satisfied:

$$
\begin{aligned}
& \frac{2 \mu(k)}{\varepsilon(k)}(\mu(k)+p(k))+\mu(k)>\frac{1}{2} \sigma_{1}^{2}(k)\left(1+b_{1}(k)\right)+\left(\frac{2 \mu(k)}{\varepsilon(k)}+\frac{3}{2}\right) \int_{U} D_{1}^{2}(k, u) v(d u), \\
& \left(\frac{2 \mu(k)}{\varepsilon(k)}+1\right)(\mu(k)+p(k))>\frac{1}{2} \sigma_{2}^{2}(k)\left(1+\frac{2 \mu(k)}{\varepsilon(k)}\right)+\left(\frac{2 \mu(k)}{\varepsilon(k)}+\frac{3}{2}\right) \int_{U} D_{2}^{2}(k, u) v(d u)
\end{aligned}
$$


and

$$
\mu(k)>\frac{1}{2} \sigma_{3}^{2}(k)+\frac{3}{2} \int_{U} D_{3}^{2}(k, u) v(d u) .
$$

Then

$$
\begin{aligned}
& \limsup _{t \rightarrow \infty} \frac{1}{t} E \int_{0}^{t}\left[\left(S(\tau)-\frac{\alpha_{4}(k)}{\alpha_{1}(k)} S_{k}^{*}\right)^{2}+\left(I(\tau)-\frac{\alpha_{5}(k)}{\alpha_{2}(k)} I_{k}^{*}\right)^{2}\right. \\
& \left.\quad+\left(V(\tau)-\frac{\mu(k)}{\alpha_{3}(k)} V_{k}^{*}\right)^{2}\right] d \tau \\
& \leq \frac{\alpha_{6}(k)}{\tilde{\alpha}(k)}
\end{aligned}
$$

where

$$
\begin{aligned}
& \alpha_{1}(k)=\frac{2 \mu(k)}{\varepsilon(k)}(\mu(k)+p(k))+\mu(k)-\frac{1}{2} \sigma_{1}^{2}(k)\left(1+b_{1}(k)\right) \\
& -\left(b_{2}(k)+\frac{3}{2}\right) \int_{U} D_{1}^{2}(k, u) v(d u), \\
& \alpha_{2}(k)=(\mu(k)+\alpha(k))\left(1+\frac{2 \mu(k)}{\varepsilon(k)}\right)-\frac{1}{2} \sigma_{2}^{2}(k)\left(1+b_{2}(k)\right) \\
& -\left(b_{2}(k)+\frac{3}{2}\right) \int_{U} D_{2}^{2}(k, u) v(d u), \\
& \alpha_{3}(k)=\mu(k)-\frac{1}{2} \sigma_{3}^{2}(k)-\frac{3}{2} \int_{U} D_{3}^{2}(k, u) v(d u) \\
& \alpha_{4}(k)=\frac{2 \mu(k)}{\varepsilon(k)}(v(k)+p(k))+\mu(k) \\
& \alpha_{5}(k)=(\mu(k)+\alpha(k))\left(1+\frac{2 \mu(k)}{\varepsilon(k)}\right), \\
& \alpha_{6}(k)=\frac{1}{\alpha_{1}(k)}\left[\frac{2 \mu(k)}{\varepsilon(k)}(\mu(k)+\alpha(k))+\mu(k)\right] \\
& \times\left[\frac{1}{2} \sigma_{1}^{2}(k)\left(1+b_{1}(k)\right)+\left(b_{2}(k)+\frac{3}{2}\right) \int_{U} D_{1}^{2}(k, u)\right]\left(S_{k}^{*}\right)^{2} \\
& +\frac{1}{\alpha_{2}(k)}(\mu(k)+\alpha(k))\left(1+\frac{2 \mu(k)}{\varepsilon(k)}\right) \\
& \times\left[\frac{1}{2} \sigma_{2}^{2}(k)\left(1+b_{2}(k)\right)+\left(b_{2}(k)+\frac{3}{2}\right) \int_{U} D_{2}^{2}(k, u) v(d u)\right]\left(I_{k}^{*}\right)^{2} \\
& +\frac{\mu(k)}{\alpha_{3}(k)}\left[\frac{1}{2} \sigma_{3}^{2}(k)+\frac{3}{2} \int_{U} D_{3}^{2}(k, u) v(d u)\right]\left(V_{k}^{*}\right)^{2}+\frac{1}{2} b_{1}(k) \sigma_{2}^{2}(k) I_{k}^{*} \\
& +b_{1}(k) I_{k}^{*} \int_{U}\left[D_{2}(k, u)-\log \left(1+D_{2}(k, u)\right)\right] v(d u), \\
& \tilde{\alpha}(k)=\min _{1 \leq i \leq 3}\left\{\alpha_{i}(k)\right\} .
\end{aligned}
$$


Proof Define the function $W: R_{+}^{3} \rightarrow R_{+}$by

$$
\begin{aligned}
W(S, I, V, k)= & b_{1}(k)\left(I-I_{k}^{*}-I_{k}^{*} \log \frac{I}{I_{k}^{*}}\right)+\frac{b_{2}(k)}{2}\left[\left(S-S_{k}^{*}\right)+\left(I-I_{k}^{*}\right)\right]^{2} \\
& +\frac{1}{2}\left[\left(S-S_{k}^{*}\right)+\left(I-I_{k}^{*}\right)+\left(V-V_{k}^{*}\right)\right]^{2},
\end{aligned}
$$

where $b_{1}(k)$ and $b_{2}(k)$ are positive constants to be determined later. Applying Itô's formula, we get

$d W(S, I, V, k)$

$$
\begin{aligned}
= & L W d t+b_{1}(k) \sigma_{2}(k)\left(I-I_{k}^{*}\right) d B_{2}(t)+b_{2}(k)\left(S-S_{k}^{*}+I-I_{k}^{*}\right)\left[\sigma_{1}(k) S d B_{1}(t)\right. \\
& \left.+\sigma_{2}(k) I d B_{2}(t)\right]+\left(S-S_{k}^{*}+I-I_{k}^{*}+V-V_{k}^{*}\right)\left[\sigma_{1}(k) S d B_{1}(t)+\sigma_{2}(k) I d B_{2}(t)\right. \\
& \left.+\sigma_{3}(k) V d B_{3}(t)\right]+b_{1}(k) \int_{U}\left[D_{2}(k, u) I-I_{k}^{*} \log \left(1+D_{2}(k, u)\right)\right] \tilde{N}(d t, d u) \\
& +\frac{b_{2}(k)}{2} \int_{U}\left[D_{1}(k, u) S+D_{2}(k, u) I\right]^{2} \widetilde{N}(d t, d u) \\
& +b_{2}(k) \int_{U}\left(S-S_{k}^{*}+I-I_{k}^{*}\right)\left[D_{1}(k, u) S+D_{2}(k, u) I\right] \tilde{N}(d t, d u) \\
& +\int_{U}\left(S-S_{k}^{*}+I-I_{k}^{*}+V-V_{k}^{*}\right)\left[D_{1}(k, u) S+D_{2}(k, u) I+D_{3}(k, u) V\right] \tilde{N}(d t, d u) \\
& +\frac{1}{2} \int_{U}\left[D_{1}(k, u) S+D_{2}(k, u) I+D_{3}(k, u) V\right]^{2} \tilde{N}(d t, d u) .
\end{aligned}
$$

In detail,

$$
\begin{aligned}
L W= & b_{1}(k) \beta(k)\left(S-S_{k}^{*}\right)\left(I-I_{k}^{*}\right)+b_{2}(k)\left(S-S_{k}^{*}+I-I_{k}^{*}\right)\left[-(\mu(k)+p(k))\left(S-S_{k}^{*}\right)\right. \\
& \left.-(\mu(k)+\alpha(k))\left(I-I_{k}^{*}\right)+\varepsilon(k)\left(V-V_{k}^{*}\right)\right]+\left(S-S_{k}^{*}+I-I_{k}^{*}+V-V_{k}^{*}\right) \\
& \times\left[-\mu(k)\left(S-S_{k}^{*}\right)-(\mu(k)+\alpha(k))\left(I-I_{k}^{*}\right)-\mu(k)\left(V-V_{k}^{*}\right)\right] \\
& +\frac{1}{2}\left(1+b_{2}(k)\right)\left(\sigma_{1}^{2}(k) S^{2}+\sigma_{2}^{2}(k) I^{2}\right)+\frac{1}{2} \sigma_{3}^{2}(k) V^{2}+\frac{1}{2} b_{1}(k) \sigma_{2}^{2}(k) I_{k}^{*} \\
& +b_{1}(k) I_{k}^{*} \int_{U}\left[D_{2}(k, u)-\log \left(1+D_{2}(k, u)\right)\right] v(d u) \\
& +\frac{1}{2} b_{2}(k) \int_{U}\left[D_{1}(k, u) S+D_{2}(k, u) I\right]^{2} v(d u) \\
& +\frac{1}{2} \int_{U}\left[D_{1}(k, u) S+D_{2}(k, u) I+D_{3}(k, u) V\right]^{2} v(d u)+\sum_{l=1}^{N} \gamma_{k l} W(S, I, V, l) \\
= & {\left[b_{2}(k)(\mu(k)+p(k))+\mu(k)\right]\left(S-S_{k}^{*}\right)^{2}-(\mu(k)+\alpha(k))\left(1+b_{2}(k)\right)\left(I-I_{k}^{*}\right)^{2} } \\
& -\mu(k)\left(V-V_{k}^{*}\right)^{2} \\
& +\left[b_{1}(k) \beta(k)-b_{2}(k)(2 \mu(k)+\alpha(k)+p(k))-(2 \mu(k)+\alpha(k))\right]\left(S-S_{k}^{*}\right)\left(I-I_{k}^{*}\right) \\
& +\left[b_{2}(k) \varepsilon(k)-2 \mu(k)\right]\left(S-S_{k}^{*}\right)\left(V-V_{k}^{*}\right) \\
& +\left[b_{2}(k) \varepsilon(k)-2 \mu(k)-\alpha(k)\right]\left(I-I_{k}^{*}\right)\left(V-V_{k}^{*}\right)
\end{aligned}
$$




$$
\begin{aligned}
& +\frac{1}{2}\left(1+b_{2}(k)\right)\left(\sigma_{1}^{2}(k) S^{2}+\sigma_{2}^{2}(k) I^{2}\right)+\frac{1}{2} \sigma_{3}^{2}(k) V^{2}+\frac{1}{2} b_{1}(k) \sigma_{2}^{2}(k) I_{k}^{*} \\
& +b_{1}(k) I_{k}^{*} \int_{U}\left[D_{2}(k, u)-\log \left(1+D_{2}(k, u)\right)\right] v(d u) \\
& +\frac{1}{2} b_{2}(k) \int\left[D_{1}(k, u) S+D_{2}(k, u) I\right]^{2} v(d u) \\
& +\frac{1}{2} \int_{U}\left[D_{1}(k, u) S+D_{2}(k, u) I+D_{3}(k, u) V\right]^{2} v(d u)+\sum_{l=1}^{N} \gamma_{k l} W(S, I, V, l) .
\end{aligned}
$$

Note that

$$
b_{2}(k) \varepsilon(k)-2 \mu(k)=0, \quad b_{1}(k) \beta(k)-b_{2}(k)(2 \mu(k)+\alpha(k)+p(k))-(2 \mu(k)+\alpha(k))=0 .
$$

Then

$$
\begin{aligned}
L W \leq & -\left[\frac{2 \mu(k)}{\varepsilon(k)}(\mu(k)+p(k))+\mu(k)\right]\left(S-S_{k}^{*}\right)^{2} \\
& +\left[\frac{1}{2} \sigma_{1}^{2}(k)\left(1+b_{1}(k)\right)+\left(b_{2}(k)+\frac{3}{2}\right) \int_{U} D_{1}^{2}(k, u) v(d u)\right] S^{2} \\
& -(\mu(k)+\alpha(k))\left(1+\frac{2 \mu(k)}{\varepsilon(k)}\right)\left(I-I_{k}^{*}\right)^{2} \\
& +\left[\frac{1}{2} \sigma_{2}^{2}(k)\left(1+b_{2}(k)\right)+\left(b_{2}(k)+\frac{3}{2}\right) \int_{U} D_{2}^{2}(k, u) v(d u)\right] I^{2} \\
& -\mu(k)\left(V-V_{k}^{*}\right)^{2}+\left[\frac{1}{2} \sigma_{3}^{2}(k)+\frac{3}{2} \int_{U} D_{3}^{2}(k, u) v(d u)\right] V^{2}+\frac{1}{2} b_{1}(k) \sigma_{2}^{2}(k) I_{k}^{*} \\
& +b_{1}(k) I_{k}^{*} \int_{U}\left[D_{2}(k, u)-\log \left(1+D_{2}(k, u)\right)\right] v(d u)+\sum_{l=1}^{N} \gamma_{k l} W(S, I, V, l) \\
\leq & -\alpha_{1}(k)\left(S-\frac{\alpha_{4}(k)}{\alpha_{1}(k)} S_{k}^{*}\right)^{2}-\alpha_{2}(k)\left(I-\frac{\alpha_{5}(k)}{\alpha_{2}(k)} I_{k}^{*}\right)^{2}-\alpha_{3}(k)\left(V-\frac{\mu(k)}{\alpha_{3}(k)} V_{k}^{*}\right)^{2} \\
& +\alpha_{6}(k)+\sum_{l=1}^{N} \gamma_{k l} W(S, I, V, l),
\end{aligned}
$$

where $\alpha_{i}(k)(i=1, \ldots, 6)$ are defined as in Theorem 5.1 .

Furthermore, we define $\breve{b}=\max \left\{\frac{b_{i}(l)}{b_{i}(k)}, i=1,2,1 \leq k, l \leq N\right\}$, and there exists a constant $\bar{B}$ such that

$$
\begin{aligned}
\sum_{l=1}^{N} \gamma_{k l} W(S, I, V, l) & \leq \check{b}\left(\sum_{l=1}^{N}\left|\gamma_{k l}\right|\right) W(S, I, V, k) \\
& :=\bar{B} W(S, I, V, k) .
\end{aligned}
$$

Integrating both sides of (5.2) from 0 to $t$ and taking expectation, we have

$$
\begin{aligned}
0 & \leq E W(S(t), I(t), V(t), r(t)) \\
& \leq W(S(0), I(0), V(0), r(0))+E \int_{0}^{t} L W d \tau
\end{aligned}
$$




$$
\begin{aligned}
= & W(S(0), I(0), V(0), r(0))-E \int_{0}^{t}\left[\alpha_{1}(k)\left(S-\frac{\alpha_{4}(k)}{\alpha_{1}(k)} S_{k}^{*}\right)^{2}+\alpha_{2}(k)\left(I-\frac{\alpha_{5}(k)}{\alpha_{2}(k)} I_{k}^{*}\right)^{2}\right. \\
& \left.+\alpha_{3}(k)\left(V-\frac{\mu(k)}{\alpha_{3}(k)} V_{k}^{*}\right)^{2}\right] d \tau+\alpha_{6}(k) t+\bar{B} E \int_{0}^{t} W(S(\tau), I(\tau), V(\tau), r(\tau)) d \tau \\
\leq & \left\{W(S(0), I(0), V(0), r(0))-E \int_{0}^{t}\left[\alpha_{1}(k)\left(S-\frac{\alpha_{4}(k)}{\alpha_{1}(k)} S_{k}^{*}\right)^{2}+\alpha_{2}(k)\left(I-\frac{\alpha_{5}(k)}{\alpha_{2}(k)} I_{k}^{*}\right)^{2}\right.\right. \\
& \left.\left.+\alpha_{3}(k)\left(V-\frac{\mu(k)}{\alpha_{3}(k)} V_{k}^{*}\right)^{2}\right] d \tau+\alpha_{6}(k) t\right\} e^{\bar{B} t} .
\end{aligned}
$$

Therefore, we get

$$
\begin{aligned}
& \limsup _{t \rightarrow \infty} \frac{1}{t} E \int_{0}^{t}\left[\left(S(\tau)-\frac{\alpha_{4}(k)}{\alpha_{1}(k)} S_{k}^{*}\right)^{2}+\left(I(\tau)-\frac{\alpha_{5}(k)}{\alpha_{2}(k)} I_{k}^{*}\right)^{2}+\left(V(\tau)-\frac{\mu(k)}{\alpha_{3}(k)} V_{k}^{*}\right)^{2}\right] d \tau \\
& \quad \leq \frac{\alpha_{6}(k)}{\tilde{\alpha}(k)}
\end{aligned}
$$

where $\tilde{\alpha}(k)=\min _{1 \leq i \leq 3}\left\{\alpha_{i}(k)\right\}$.

The theorem is proved.

Remark 5.1 According to Theorem 5.1, we obtain that the solution of model (1.3) fluctuates around a certain level relevant to $E_{k}^{*}\left(\frac{\alpha_{4}(k)}{\alpha_{1}(k)} S_{k}^{*}, \frac{\alpha_{5}(k)}{\alpha_{2}(k)} I_{k}^{*}, \frac{\mu(k)}{\alpha_{3}(k)} V_{k}^{*}\right), \sigma_{i}(k)(i=1,2,3)$, and $D_{i}(k, u)(i=1,2,3)$. With the values of $\sigma_{i}(k)$ and $D_{i}(k, u)(i=1,2,3)$ decreasing, the solution of system (1.3) will be close to $E_{k}^{*}$, and the difference between $(S(t), I(t), V(t))$ and $E_{k}^{*}$ will also decrease. Besides, if $D_{i}(k, u)=0(i=1,2,3)$, Theorem 5.1 shows that the solution of the stochastic regime switching SIS model with vaccination will fluctuate around a certain level relevant to $E_{k}^{*}$ and $\sigma_{i}(k)(i=1,2,3)$.

\section{Conclusions}

We present a stochastic regime switching SIS epidemic model with vaccination driven by a Lévy noise. Based on this model, we analyze the existence and uniqueness of its global positive solution. We also discuss the asymptotic behavior of the solution to this model around the disease-free equilibrium $P_{k}^{0}$ and the endemic equilibrium $P_{k}^{*}$.

The asymptotic behavior of solutions to SDEs is very important. From the view of applications, investigating the stability in distribution is a more interesting question in stochastic population systems. Bao et al. $[15,16]$ did a pioneering work in this area. After that, many results on the stochastic population models with jumps have been reported [17, 18, 26-29].

\section{Acknowledgements}

The author thanks Prof. Y Li for his valuable discussion. We would like to thank the editor and anonymous referees for their valuable comments and suggestions on this paper. The work is supported by the Department of Education of Jilin Province 13th Five-Year Plan to support scientific research projects (JJKH20170025KJ).

\section{Competing interests}

The author declares that they have no competing interests. 


\section{Publisher's Note}

Springer Nature remains neutral with regard to jurisdictional claims in published maps and institutional affiliations.

\section{Received: 8 August 2017 Accepted: 12 November 2017 Published online: 01 December 2017}

\section{References}

1. Chen, FH: A susceptible-infected epidemic model with voluntary vaccinations. J. Math. Biol. 53(2), 253-272 (2006)

2. Shim, E, Feng, Z, Martcheva, M, Castillo-Chavez, C: An age-structured epidemic model of rotavirus with vaccination. J. Math. Biol. 53(4), 719-746 (2006)

3. Moneim, I, Greenhalgh, D: Threshold and stability results for an sirs epidemic model with a general periodic vaccination strategy. J. Biomath. Syst. 13, 131-150 (2005)

4. Greenhalgh, D: Hopf bifurcation in epidemic models with a latent period and nonpermanent immunity. Math. Comput. Model. 25(2), 85-107 (1997)

5. Li, J, Ma, Z: Qualitative analyses of SIS epidemic model with vaccination and varying total population size. Math Comput. Model. 35(11-12), 1235-1243 (2002)

6. Li, J, Ma, Z: Global analysis of SIS epidemic models with variable total population size. Math. Comput. Model. 39(11-12), 1231-1242 (2004)

7. Vicence, $M$, Daniel, $C$, Werner, $H$ : Stochastic fluctuations of the transmission rate in the susceptible-infected-susceptible epidemic model. Phys. Rev. E (2012)

8. Chen, C, Kang, Y: The asymptotic behavior of a stochastic vaccination model with backward bifurcation. Appl. Math. Model. (2016)

9. Zhao, YN, Jiang, DQ: Dynamics of stochastically perturbed SIS epidemic model with vaccination. Abstr. Appl. Anal. 2013, Article ID 517439 (2013)

10. Applebaum, D: Lévy Process and Stochastic Calculus, 2nd edn. Cambridge University Press, Cambridge (2009)

11. Pasel, A, Kosko, B: Stochastic resonance in continuous and spiking neuron models with Lévy noise. IEEE Trans. Neural Netw. 19(12), 1993-2008 (2008)

12. $\mathrm{Xu}, \mathrm{Y}, \mathrm{Wang}, \mathrm{X}, \mathrm{Zhang}, \mathrm{H}, \mathrm{Xu}, \mathrm{W}$ : Stochastic stability for nonlinear systems driven by Lévy noise. Nonlinear Dyn. 68 , 7-15 (2012)

13. Liu, Q: Asymptotic properties of a stochastic $n$-species Gilpin-Ayala competitive model with Lévy jumps and Markovian switching. Commun. Nonlinear Sci. Numer. Simul. 26, 1-10 (2015)

14. Luo, Q, Mao, XR: Stochastic population dynamics under regimes switching. J. Math. Anal. Appl. 334, 69-84 (2007)

15. Bao, J, Mao, X, Yin, G, Yuan, C: Competitive Lotka-Volterra population dynamics with jumps. Nonlinear Anal. 74, $6601-6616(2011)$

16. Bao, J, Yuan, C: Stochastic population dynamics driven by Lévy noise. J. Math. Anal. Appl. 391, 363-375 (2012)

17. Liu, M, Wang, K: Dynamics of a Leslie-Gower Holling-type II predator-prey system with Lévy jumps. Nonlinear Anal. $85,204-213(2013)$

18. Liu, M, Wang, K: Stochastic Lotka-Volterra systems with Lévy noise. J. Math. Anal. Appl. 410, 750-763 (2014)

19. Zhang, X, Wang, K: Stochastic SIR model with jumps. Appl. Math. Lett. 26, 867-874 (2013)

20. Zhang, $X$, Wang, K: Stochastic SEIR model with jumps. Appl. Math. Comput. 239, 133-143 (2014)

21. Zhou, Y, Yuan, S, Zhao, D: Threshold behavior of a stochastic SIS model with Lévy jumps. Appl. Math. Comput. 275, 255-267 (2016)

22. Zhou, Y, Zhang, W: Threshold of a stochastic SIR epidemic model with Lévy jumps. Phys. A, Stat. Mech. Appl. 446 204-216 (2016)

23. Chen, C, Kang, Y: Dynamics of a stochastic multi-strain SIS epidemic model driven by Lévy noise. Commun. Nonlinear Sci. Numer. Simul. 42, 379-395 (2017)

24. Settai, A, Lahrouz, A: Stationary distribution of stochastic population systems under regime switching. Appl. Math Comput. 244, 235-243 (2014)

25. Li, D, Cui, J, Liu, M, Liu, S: The evolutionary dynamics of stochastic epidemic model with nonlinear incidence rate. Bull. Math. Biol. 77, 1705-1743 (2015)

26. Zhang, XH, Wang, K: Stability analysis of a stochastic Gilpin-Ayala model driven by Lévy noise. Commun. Nonlinear Sci. Numer. Simul. 19, 1391-1399 (2014)

27. Wu, RH, Zou, XL, Wang, K: Asymptotic properties of stochastic hybrid Gilpin-Ayala system with jumps. Appl. Math Comput. 249, 53-66 (2014)

28. Liu, M, Fan, M: Stability in distribution of a three-species stochastic cascade predator-prey system with time delays. IMA J. Appl. Math. 82(2), 396-423 (2017)

29. $\mathrm{Wu}, \mathrm{RH}, \mathrm{Zou}, \mathrm{XL}, \mathrm{Wang}, \mathrm{K}$ : Dynamics of logistic systems driven by Lévy noise under regime switching. Electron. J. Differ. Equ. 2014(76), 1 (2014) 